\title{
Stem Cell Therapy for the Treatment of Myocardial Infarction: How Far Are We Now?
}

Rinky A. Botleroo ${ }^{1}$, Renu Bhandari ${ }^{1,2}$, Rowan Ahmed ${ }^{1}$, Roaa Kareem ${ }^{1}$, Mallika Gyawali ${ }^{1}$, Nanditha Venkatesan $^{3,1}$, Opemipo D. Ogeyingbo ${ }^{1,4,5}$, Abeer O. Elshaikh ${ }^{1}$

1. Internal Medicine, California Institute of Behavioral Neurosciences \& Psychology, Fairfield, USA 2. Internal Medicine, Manipal College of Medical Sciences, Pokhara, NPL 3. Internal Medicine, All India Institute of Medical Sciences, Raipur, IND 4. Internal Medicine, Saint James School of Medicine, Park Ridge, USA 5. Public Health, Walden University, Minneapolis, USA

Corresponding author: Rinky A. Botleroo, rinkyagnes@gmail.com

\begin{abstract}
Myocardial infarction is one of the leading causes of death worldwide. Poor functional recovery of the myocardium is noticed after an event of myocardial infarction. Researchers and clinicians around the world have been engaged to regenerate the damaged human heart for a long time. Stem cell therapy is an exciting newer therapy to treat cardiovascular diseases.
\end{abstract}

Various types of stem cells have been used to revive the damaged myocardium after myocardial infarction, and they have overall demonstrated safety and moderate efficacy. The specific mechanisms by which these cells help in improving cardiac function are still not completely known. There is growing evidence that intracoronary bone marrow cell transplantation in patients with myocardial infarction beneficially affects the remodeling of the damaged myocardium.

Our systematic review article aims to assess the effects and the future of stem cell therapy in patients with myocardial Infarction. We searched articles in PubMed, ScienceDirect, and Google Scholar. Thirty-one studies that included 2171 patients in total were analyzed. Most of these studies showed stem cell therapy is safe and well tolerated in patients, and modest improvements are seen in left ventricular functions with no major adverse effects. However, some studies showed no positive and clinically significant outcomes. So, more high-quality studies on a larger scale are required to support and confirm its efficacy in remodeling damaged myocardium after myocardial infarction. We should also perform studies to determine the timing of cell delivery that is best suited for stem cell therapy.

Categories: Cardiology, Internal Medicine, Transplantation

Keywords: stem cells, myocardial infarction, stem cell therapy, bone marrow mononuclear cells, stemi, stem cell transplantation

Received 07/11/2021 Review began 07/17/2021 Review ended 08/09/2021 Published 08/09/2021

๑) Copyright 2021 Botleroo et al. This is an open access article distributed under the terms of the Creative Commons Attribution License CC-BY 4.0., which permits unrestricted use, distribution, and reproduction in any medium, provided the original author and source are credited.

\section{Introduction And Background}

One of the most common causes of morbidity and mortality globally is cardiovascular disease [1]. The oneyear mortality is approximately $13 \%$ and the five-year prognosis for patients with heart failure is $50 \%$, even though there has been tremendous advancement in the treatment of acute myocardial infarction (MI) [1].

The presence of any obstruction in the coronary arteries gives rise to acute myocardial ischemia [1]. Rupture of plaques, fissuring, or formation of any superimposed thrombus may be responsible for this obstruction formation [1]. Although there have been major advancements in the management of acute Myocardial Infarction including fibrinolysis and rapid revascularization, the prognosis remains poor due to the lack of self-repairing of the already damaged myocardium, which may result in complications like heart failure [1].

There are multiple methods to repair the damaged heart that include cell transplantation, gene therapy, stimulating innate repair pathways, direct reprogramming of cells, cardiac tissue engineering, and biomaterial delivery [2]. Among these, the most accepted strategy for heart repair is the delivery of exogenous cells [2]. Almost every cell type we can think of, such as skeletal myoblasts to pluripotent stem cells and their derivatives has been transplanted into the injured myocardium [2].

Stem cells are unspecialized immature cells that can divide and replicate themselves throughout the entire life of an organism [3]. Skeletal myoblasts (satellite cells) are classically the stem cell population within the non-cardiac musculature [4]. There are $2 \%$ to $7 \%$ improvements in ejection fractions (EF) with the administration of adult bone marrow cells (BMC) [4]. The exact mechanisms of improvement of damaged heart function by cell therapy are unclear, but it is assumed that the paracrine effect plays a central role [5]. Transplanted mesenchymal stem cells (MSCs) can engraft and differentiate into cardiomyocyte-like and endothelial cells and recruit endogenous cardiac stem cells [6]. 


\section{Cureus}

As the viability and function of autologous adult stem cells decline with age, especially in patients with MI, alternative sources of stem cells such as Wharton's jelly-derived mesenchymal stem cells (WJ-MSCs), cardiac progenitor cells can also be used [6]. Isolating and expanding resident cardiac progenitor cells present in the adult myocardium cells is a tough task. However, these are more beneficial than the other stem cell types because they are likely predestined to cardiovascular fate [5].

The first-ever encouraging study showing positive outcomes in MI patients with stem cell therapy was published by Strauer et al. in 2002, many other trials have been conducted since then [7]. The main objective of our article is to evaluate the safety and effects of transplanting stem cells in patients with acute myocardial infarction. Figure 1 given below illustrates the pathophysiology of MI.
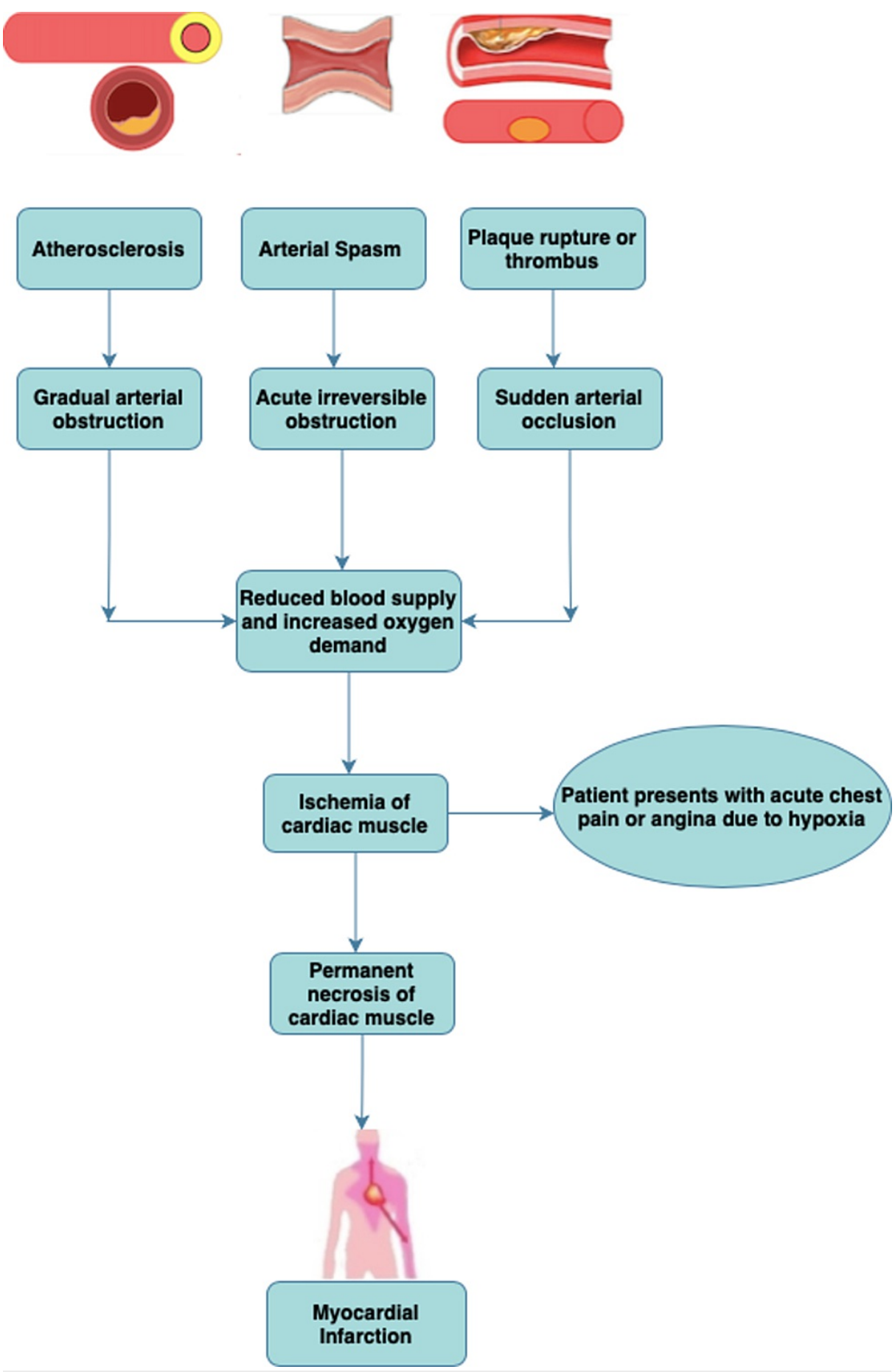

FIGURE 1: Pathophysiology of myocardial infarction

Review 


\section{Cureus}

\section{Methods}

Study Protocol

We implemented Preferred Reporting Items for Systematic Review and Meta-Analyses (PRISMA) 2020 Guidelines in our study process.

Sources of Data and Search Strategy

Articles were searched from three databases PubMed, Science Direct, and Google Scholar using specific keywords related to the research topic. The keywords used are as follows: myocardial infarction, STelevation myocardial infarction, non-ST-elevation myocardial infarction, stem cell transplantation, mononuclear bone marrow cell transplantation, adult population.

The Medical Subject Headings (MeSH) is the National Library of Medicine (NLM) controlled vocabulary thesaurus that we specifically used to search articles in the PubMed database. We performed a search using a combination of MeSH terms and text words given below. The final MeSH search strategy used was as follows:

Myocardial Infarction OR ST-Elevation Myocardial Infarction OR Non-ST-Elevation Myocardial Infarction OR Acute Myocardial Infarction OR ( "Myocardial Infarction/drug therapy"[Mesh] OR "Myocardial Infarction/mortality"[Mesh] OR "Myocardial Infarction/prevention and control"[Mesh] OR "Myocardial Infarction/therapy"[Mesh] ) AND Stem Cell Transplantation OR Stem cell therapy OR mesenchymal stem cell OR Progenitor Cell OR mononuclear bone marrow cell transplantation OR ( "Stem Cell Transplantation/instrumentation"[Mesh] OR "Stem Cell Transplantation/methods"[Mesh] OR "Stem Cell Transplantation/mortality"[Mesh] OR "Stem Cell Transplantation/therapeutic use"[Mesh] OR "Stem Cell Transplantation/therapy"[Mesh] )AND Adult.

Other databases and the keywords used for the search are mentioned in Table 1 .

\begin{tabular}{|c|c|c|c|}
\hline \multirow{2}{*}{ Databases } & \multirow{2}{*}{ Keywords used for the search } & \multicolumn{2}{|c|}{ Search results } \\
\hline & & $\begin{array}{l}\text { Initial } \\
\text { results }\end{array}$ & $\begin{array}{l}\text { Timeframe 2011- } \\
2021\end{array}$ \\
\hline PubMed & The final MeSH search strategy as mentioned above & 107,272 & 57,904 \\
\hline ScienceDirect & Myocardial Infarction and Stem cell therapy & 22,211 & 12,640 \\
\hline $\begin{array}{l}\text { Google } \\
\text { Scholar }\end{array}$ & $\begin{array}{l}\text { "Myocardial infarction" and "stem cell therapy" and "mononuclear bone marrow cell } \\
\text { transplantation" }\end{array}$ & 1,300 & 547 \\
\hline
\end{tabular}

TABLE 1: Databases and search results

Inclusion and Exclusion Criteria

We only included the articles published in the English language, which were human studies and clinical trials. We selected articles published from 2011-2021. The inclusion criteria were: (1) Patients diagnosed with myocardial infarction; (2) Patients who received stem cell therapy after myocardial infarction; (3) Age of the patients 19 and above; (4) Both male and female patients were selected. Exclusion criteria were studies on animals, reviews, or studies for which the full text was unavailable or only abstracts were available. We did not include gray literature.

Risk and Quality Assessment

Two reviewers independently (RAB and RB) extracted and evaluated the quality of the included 31 studies. Revised Cochrane's risk of bias assessment tool was used for randomized controlled trials (RCTs) and clinical trials.

Data Extraction

Two reviewers (RAB and RB) separately extracted relevant data from included 31 studies using standard data extraction forms and data was extracted under the following headings: name of the author, country of the study, the name of the journal where it was published, year of publication, study design, the title of the study, sample size, patient characteristics, size of the treatment group and control group, follow-up period, 


\section{Cureus}

and outcome of the study.

Results

A total of 2650 articles from PubMed, 221 articles from ScienceDirect, and 547 articles from Google Scholar were collected using the search strategy we have mentioned in the method section and were then screened based on the title and abstract related to our study. We also removed the duplicates. Then, we filtered out a few papers based on the eligibility criteria and availability of full text. In the end, only 31 items were included, and these articles were checked for quality based on their study characteristics. A complete Preferred Reporting Items for Systematic Review and Meta-Analyses (PRISMA) flow diagram is given below in Figure 2.

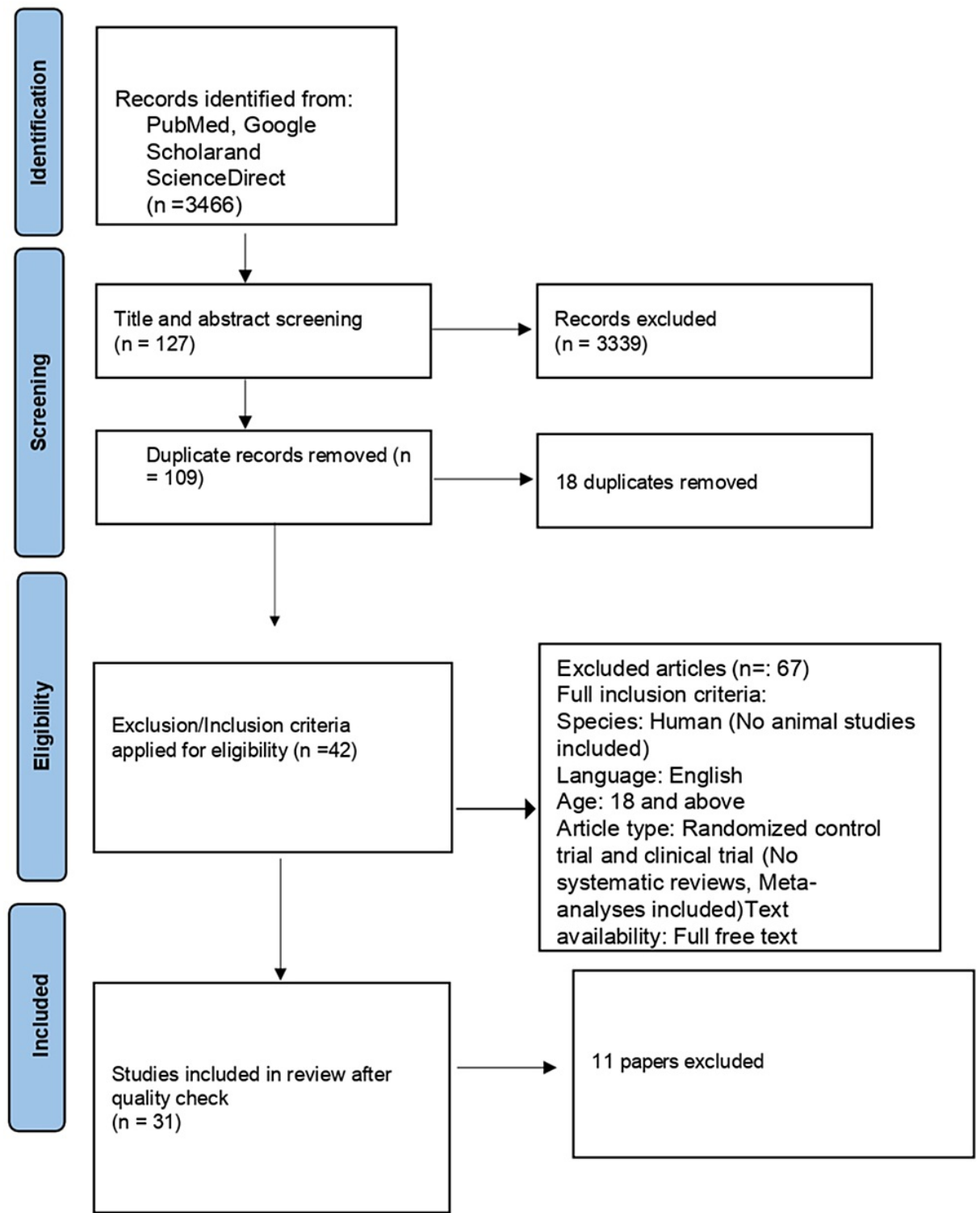

FIGURE 2: PRISMA flow diagram

PRISMA: Preferred Reporting Items for Systematic Review and Meta-Analyses

Study Characteristics

Our systematic review includes patients from 31 studies. Table 2 shows the characteristics of the included studies and the outcomes of the studies. 


\section{Cureus}

\begin{tabular}{|c|c|c|c|c|c|c|c|c|}
\hline & & design & & size & & & & \\
\hline $\begin{array}{l}\text { Benedek et } \\
\text { al. [8] }\end{array}$ & Romania / 2014 & ССT & $\begin{array}{l}\text { Adult with } \mathrm{h} / \mathrm{o} \text { STEMI and PPCI } \\
\text { and abnormalities in wall } \\
\text { motion and less than } 50 \% \\
\text { stenosis }\end{array}$ & 18 & Autologous MNC $=9$ & Placebo $=9$ & $\begin{array}{l}\text { Four years with } \\
\text { clinical } \\
\text { examinations, } \\
\text { ECG, } \\
\text { Echocardiography, } \\
\text { 64-slice CCTA }\end{array}$ & $\begin{array}{l}\text { A small improvement in EF } \\
\text { and the plaque burden is } \\
\text { lower in coronary segments } \\
\text { treated with stem cells }\end{array}$ \\
\hline $\begin{array}{l}\text { Alestalo et al. } \\
\text { [9] }\end{array}$ & Finland / 2015 & $\begin{array}{l}\text { Double- } \\
\text { blinded } \\
\text { RCT }\end{array}$ & $\begin{array}{l}\text { H/o STEMI, }<75 \text { years, } \\
\text { hemodynamically stable and no } \\
\text { cardiogenic shock or rescue } \\
\text { PCI/CABG }\end{array}$ & 26 & $\mathrm{BMMNC}=14$ & Placebo $=12$ & $\begin{array}{l}\text { Cytokines after } \\
\text { four days and LV } \\
\text { angiogram after } \\
\text { six months }\end{array}$ & $\begin{array}{l}\text { A balancing effect between } \\
\text { the anti-inflammatory and } \\
\text { proinflammatory cytokine } \\
\text { BMMNC group at day four }\end{array}$ \\
\hline $\begin{array}{l}\text { Bozdag- } \\
\text { Turan et al. } \\
{[10]}\end{array}$ & $\begin{array}{l}\text { Germany / } \\
2012\end{array}$ & СCT & $\begin{array}{l}\text { 18-80 years with } \mathrm{h} / \mathrm{o} \text { STEMI, } \\
\text { absence of co-morbidities, } \\
\text { cancer, and active bleeding or } \\
\text { trauma in the last two months }\end{array}$ & 24 & $\mathrm{BMC}=12$ & Placebo $=12$ & $\begin{array}{l}\text { Six months with } \\
\text { left } \\
\text { ventriculography }\end{array}$ & $\begin{array}{l}\text { Infarct size and BNP } \\
\text { level decreased, and global } \\
\text { EF and infarct wall movement } \\
\text { velocity were increased in the } \\
\text { stem cell group }\end{array}$ \\
\hline $\begin{array}{l}\text { Choudry et al. } \\
\text { [11] }\end{array}$ & $\begin{array}{l}5 \text { centers in } \\
\text { Europe [United } \\
\text { Kingdom (3); } \\
\text { Switzerland (1); } \\
\text { Denmark (1)] / } \\
2016\end{array}$ & $\begin{array}{l}\text { Double- } \\
\text { blinded } \\
\text { RCT }\end{array}$ & $\begin{array}{l}\text { Acute anterior MI with anterior } \\
\text { wall motion abnormality and } \mathrm{h} / \mathrm{o} \\
\mathrm{PPCl}\end{array}$ & 100 & $\mathrm{BMMNC}=55$ & Placebo $=45$ & $\begin{array}{l}\text { One year with } \\
\text { cardiac MRI and } \\
\text { Echocardiography }\end{array}$ & $\begin{array}{l}\text { Small non-significant } \\
\text { improvement in LVEF }\end{array}$ \\
\hline $\begin{array}{l}\text { Duan et al. } \\
\text { [12] }\end{array}$ & China / 2015 & RCT & $\begin{array}{l}\text { H/O MI, }<75 \text { years with planned } \\
\text { CABG for triple-vessel disease, } \\
\text { LVEF }<30 \% \text {, and no aneurysm } \\
\text { or valvular diseases }\end{array}$ & 42 & $\mathrm{CABG}+\mathrm{BMMNC}=24$ & $\begin{array}{l}\text { Only CABG = } \\
18\end{array}$ & $\begin{array}{l}\text { One year with } \\
\text { Echocardiography }\end{array}$ & $\begin{array}{l}\text { Improvement in left } \\
\text { ventricular functions in the } \\
\text { treatment group }\end{array}$ \\
\hline Gao et al. [6] & China / 2015 & $\begin{array}{l}\text { Double- } \\
\text { blinded } \\
\text { RCT }\end{array}$ & $\begin{array}{l}\text { 18-80 years with a h/o STEMI } \\
\text { and reperfusion with stent } \\
\text { implantation and LV local wall- } \\
\text { motion abnormality. CK > three- } \\
\text { fold the upper limit of the } \\
\text { normal }\end{array}$ & 116 & WJ-MSC = 58 & Placebo $=58$ & $\begin{array}{l}18 \text { months with F- } \\
\text { 18-FDG-PET and } \\
\text { 99mTC-SPECT and } \\
\text { two-dimensional } \\
\text { Echocardiography }\end{array}$ & $\begin{array}{l}\text { LVEF significantly increased } \\
\text { and LVESV and LVEDV } \\
\text { greatly decreased in the } \\
\text { treatment group }\end{array}$ \\
\hline Hu et al. [13] & China / 2015 & RCT & $\begin{array}{l}\text { 18-75 years old with acute } \\
\text { STEMI and PPCI with stent } \\
\text { implantation or thrombolysis } \\
\text { and LV local wall motion } \\
\text { abnormality }\end{array}$ & 36 & $\begin{array}{l}\text { N-BMCs = 11, HP-BMCs = } \\
11\end{array}$ & $\begin{array}{l}\text { Standard } \\
\text { therapy }=14\end{array}$ & Six and 12 months & $\begin{array}{l}\text { Improvement in changes of } \\
\text { LVEDV, LVESV, and WMSI in } \\
\text { the HP-BMC group. } \\
\text { Myocardial perfusion defect } \\
\text { ratio was reduced in HP- } \\
\text { BMCs and N-BMC groups at } \\
\text { six months }\end{array}$ \\
\hline $\begin{array}{l}\text { Huan et al. } \\
{[14]}\end{array}$ & China / 2015 & СT & $\begin{array}{l}18-75 \text { years old with a h/o acute } \\
\text { STEMI and treatment with PCI, } \\
\text { LVEF }<50 \%\end{array}$ & 104 & $\begin{array}{l}\text { Group } \mathrm{A}=\mathrm{BMMNC} \text { within } \\
\text { two hours after } \mathrm{PCl}=27, \\
\text { Group } \mathrm{B}=\text { three-seven } \\
\text { days after } \mathrm{PCl}=26 \text {, Group } \\
\mathrm{C}=\text { seven to } 30 \text { days after } \\
\mathrm{PCl}=26\end{array}$ & $\begin{array}{l}\text { Placebo }=25 \\
\text { patients }\end{array}$ & $\begin{array}{l}\text { Six months with } \\
\text { angiography. } \\
\text { SPECT and } \\
\text { Echocardiography } \\
\text { at six and } 12 \\
\text { months }\end{array}$ & $\begin{array}{l}\text { Effects of cell therapy given } \\
\text { within } 24 \text { hours are the same } \\
\text { as to given three-seven days } \\
\text { after PPCl }\end{array}$ \\
\hline Kim et al. [15] & $\begin{array}{l}\text { South Korea / } \\
2018\end{array}$ & RCT & STEMI & 26 & BMMNC $=14$ & Placebo = 12 & $\begin{array}{l}\text { Four and 12- } \\
\text { months with } \\
\text { SPECT }\end{array}$ & $\begin{array}{l}\text { Increase in the LVEF from } \\
\text { baseline to the fourth month } \\
\text { and twelfth month in the bone } \\
\text { marrow mesenchymal stem } \\
\text { cells group. }\end{array}$ \\
\hline Lee et al. [16] & Korea / 2014 & СT & 18-70 years old with STEMI & 69 & $\mathrm{MSC}=33$ & Placebo = 36 & $\begin{array}{l}\text { Six months with } \\
\text { SPECT }\end{array}$ & $\begin{array}{l}\text { Safe with modest } \\
\text { improvement in LVEF }\end{array}$ \\
\hline $\begin{array}{l}\text { Makkar et al. } \\
\text { [17] }\end{array}$ & USA / 2012 & СT & $\begin{array}{l}\mathrm{H} / \mathrm{o} \text { two to four weeks of MI and } \\
\mathrm{LVEF}=25-45 \%\end{array}$ & 25 & $\begin{array}{l}\text { Cardio sphere-derived } \\
\text { autologous stem cells }=17\end{array}$ & $\begin{array}{l}\text { Standard } \\
\text { therapy }=8\end{array}$ & $\begin{array}{l}\text { Six months with } \\
\text { MRI }\end{array}$ & $\begin{array}{l}\text { Scar mass was reduced, } \\
\text { increase in viable heart mass } \\
\text { and regional contractility in } \\
\text { the CDC group. LVEDV, } \\
\text { LVESV, and LVEF were the } \\
\text { same in the two groups }\end{array}$ \\
\hline
\end{tabular}




\section{Cureus}

\begin{tabular}{|c|c|c|c|c|c|c|c|c|}
\hline $\begin{array}{l}\text { Malliaras et } \\
\text { al. [18] }\end{array}$ & USA / 2014 & RCT & $\begin{array}{l}\text { Patients of CADUCEUS trial } \\
\text { were followed up for a year }\end{array}$ & 25 & $\begin{array}{l}\text { Cardio sphere-derived } \\
\text { autologous stem cells = } 17\end{array}$ & $\begin{array}{l}\text { Standard } \\
\text { therapy }=8\end{array}$ & One year with MRI & $\begin{array}{l}\text { Scar size reduced, increased } \\
\text { viable myocardium, and } \\
\text { improved regional function of } \\
\text { infarcted myocardium }\end{array}$ \\
\hline $\begin{array}{l}\text { Micheu et al. } \\
\text { [19] }\end{array}$ & Romania / 2015 & CT & $\begin{array}{l}\text { 18-81 years old with STEMI \& } \\
\text { h/o angioplasty with stent } \\
\text { implantation, LVEF }<40 \% \text {. }\end{array}$ & 18 & Autologous BMCs = 7 & $\begin{array}{l}\text { Standard } \\
\text { therapy }=11\end{array}$ & $\begin{array}{l}\text { Six months with } \\
\text { clinical } \\
\text { examination, } \\
\text { Echocardiography, } \\
24 \text { hours ECG }\end{array}$ & $\begin{array}{l}\text { Safe and LVEF was } \\
\text { increased }\end{array}$ \\
\hline $\begin{array}{l}\text { Moccetti et } \\
\text { al. [20] }\end{array}$ & $\begin{array}{l}\text { Switzerland / } \\
2012\end{array}$ & СT & $\begin{array}{l}\text { Acute anterior STEMI treated by } \\
\mathrm{PPCl} \text { and } \mathrm{LVEF}<50 \% \text {. }\end{array}$ & 60 & Autologous BMMNC $=23$ & $\begin{array}{l}\text { Standard } \\
\text { therapy }=37\end{array}$ & $\begin{array}{l}\text { Five years with } \\
\text { Echocardiography }\end{array}$ & $\begin{array}{l}\text { Safe and LV function } \\
\text { improved }\end{array}$ \\
\hline $\begin{array}{l}\text { Moreira et al. } \\
\text { [21] }\end{array}$ & Brazil / 2011 & RCT & $\begin{array}{l}18-80 \text { years old with } \mathrm{h} / \mathrm{o} \mathrm{MI} \text { and } \\
\text { reperfusion and involving more } \\
\text { than } 10 \% \text { of the } \mathrm{LV}\end{array}$ & 30 & $\begin{array}{l}\text { BMMNC via anterograde } \\
\text { intra-arterial coronary (IAC) } \\
\text { delivery = } 14, \text { BMMNC via } \\
\text { retrograde intravenous } \\
\text { coronary (IVC) delivery = } \\
10\end{array}$ & Placebo $=6$ & $\begin{array}{l}\text { Cardiac MRI was } \\
\text { performed before } \\
\text { cell injection }\end{array}$ & $\begin{array}{l}\text { The retrograde approach to } \\
\text { deliver stem cells was safe } \\
\text { and cell retention by cardiac } \\
\text { tissue is more in the } \\
\text { anterograde approach }\end{array}$ \\
\hline $\begin{array}{l}\text { Nair et al. } \\
\text { [22] }\end{array}$ & India / 2015 & $\mathrm{RCT}$ & $\begin{array}{l}\text { Anterior MI and LVEF }=20-50 \% \text {, } \\
20-65 \text { years with } \mathrm{h} / \mathrm{O} \text { CAG } \\
\text { between one to three weeks }\end{array}$ & 250 & $\begin{array}{l}\text { Stem cell therapy }+ \\
\text { standard care }=125\end{array}$ & $\begin{array}{l}\text { Standard care } \\
=125\end{array}$ & $\begin{array}{l}\text { Six months with } \\
\text { Echocardiography }\end{array}$ & $\begin{array}{l}\text { Safe, but not clinically } \\
\text { significant }\end{array}$ \\
\hline $\begin{array}{l}\text { Naseri et al. } \\
\text { [23] }\end{array}$ & Iran / 2018 & RCT & $\begin{array}{l}\text { 18-75 years old with a } \mathrm{h} / \mathrm{o} \text { acute } \\
\text { MI infarction, eligible for elective } \\
\text { CABG }\end{array}$ & 77 & $\operatorname{CD} 133(+)=21, \mathrm{MNC}=30$ & Placebo $=26$ & $\begin{array}{l}\text { Six and } 18 \text { months } \\
\text { after CABG with } \\
\text { SPECT }\end{array}$ & $\begin{array}{l}\text { Significant differences were } \\
\text { seen between the MNC and } \\
\text { placebo groups in LVEF and } \\
\text { a decrease in the LV } \\
\text { thickening }\end{array}$ \\
\hline $\begin{array}{l}\text { Nicolau et al. } \\
{[24]}\end{array}$ & Brazil / 2018 & $\mathrm{RCT}$ & $\begin{array}{l}30-80 \text { years, LVEF } \leq 50 \% \text {, and } \\
\text { regional dysfunction in the } \\
\text { infarct-related area }\end{array}$ & 120 & BMMNC $=66$ & Placebo $=55$ & $\begin{array}{l}\text { Six months with } \\
\text { MRI }\end{array}$ & No significant effects \\
\hline $\begin{array}{l}\text { Peregud- } \\
\text { Pogorzelska } \\
\text { et al. [25] }\end{array}$ & Poland / 2020 & $\begin{array}{l}\text { CCT not } \\
\text { randomized }\end{array}$ & $\begin{array}{l}<65 \text { years old with first } \mathrm{Ml} \text { and } \\
\mathrm{EF} \leq 45 \%\end{array}$ & 34 & $\begin{array}{l}\text { Standard therapy + } \\
\text { autologous BM-derived } \\
\text { LIN- SPCs = } 15\end{array}$ & $\begin{array}{l}\text { Only standard } \\
\text { therapy }=19\end{array}$ & $\begin{array}{l}\text { One, three, six } \\
\text { months, and one } \\
\text { year with } \\
\text { Echocardiography }\end{array}$ & $\begin{array}{l}\text { Safe and }>10 \% \text { improvement } \\
\text { in LVEF is noticed at } 12 \\
\text { months }\end{array}$ \\
\hline $\begin{array}{l}\text { Quyyumi et } \\
\text { al. [26] }\end{array}$ & USA / 2017 & RCT & $\begin{array}{l}\text { STEMI with a stent and LVEF } \leq \\
48 \% \text { and } \geq \text { four days post stent }\end{array}$ & 161 & $\begin{array}{l}\text { Intracoronary infusion of } \\
\text { autologous } \mathrm{CD} 34(+) \text { cell }= \\
78\end{array}$ & Placebo $=83$ & $\begin{array}{l}\text { Six months with } \\
\text { SPECT }\end{array}$ & Safe \\
\hline $\begin{array}{l}\text { Rodrigo et al. } \\
\text { [27] }\end{array}$ & $\begin{array}{l}\text { Netherlands / } \\
2013\end{array}$ & ССT & $\begin{array}{l}\text { First acute STEMI treated with } \\
\text { PPCI and maximum CK level } \\
\text { was }>1,600 \mathrm{U} / \mathrm{L}\end{array}$ & 54 & $M S C=9$ & $\begin{array}{l}\text { Standard } \\
\text { therapy }=45 \\
\text { matched but } \\
\text { nonrandomized } \\
\text { patients }\end{array}$ & $\begin{array}{l}\text { Three, six months, } \\
\text { one year, four-five } \\
\text { years with } \\
\text { Echocardiography, } \\
\text { Holter, and clinical } \\
\text { examination }\end{array}$ & $\begin{array}{l}\text { Improvements in LV function } \\
\text { but not significantly different } \\
\text { when compared to controls }\end{array}$ \\
\hline $\begin{array}{l}\text { Roncalli et al. } \\
\text { [28] }\end{array}$ & France / 2011 & RCT & $\begin{array}{l}\text { Acute } \mathrm{MI} \text { and successful } \\
\text { reperfusion with } \mathrm{LVEF} \leq 45 \% \text {, } \\
\text { age } 18-75 \text { years }\end{array}$ & 101 & BMMNC $=52$ & Placebo $=49$ & $\begin{array}{l}\text { Three months with } \\
\text { MRI, } \\
\text { Echocardiography, } \\
\text { and SPECT }\end{array}$ & $\begin{array}{l}\text { Multivariate analysis shows } \\
\text { improvement of myocardial } \\
\text { viability than univariate } \\
\text { analysis }\end{array}$ \\
\hline $\begin{array}{l}\text { San Roman } \\
\text { et al. [29] }\end{array}$ & Spain / 2015 & RCT & $\begin{array}{l}\text { Adult, acute } \mathrm{MI} \text { with } \mathrm{PPCl} \text { or } \\
\text { post-fibrinolysis } \mathrm{PCl} \text { and } \\
\text { rapamycin drug-eluting stent } \\
\text { implantation }\end{array}$ & 120 & $\begin{array}{l}\text { BMMNC }=30, \mathrm{GCSF}=30, \\
\mathrm{G}-\mathrm{CSF}+\text { cells }=29\end{array}$ & $\begin{array}{l}\text { Standard } \\
\text { therapy }=31\end{array}$ & $\begin{array}{l}12 \text { months with } \\
\text { cardiac MRI }\end{array}$ & $\begin{array}{l}\text { Not many differences among } \\
\text { the four groups }\end{array}$ \\
\hline Shah et al. [3] & India / 2014 & CT & $\begin{array}{l}\text { 30-70 years old, acute MI } \\
\text { with } \mathrm{PCl}\end{array}$ & 19 & Autologous BMCS $=12$ & $\begin{array}{l}\text { Standard } \\
\text { therapy }=7\end{array}$ & $\begin{array}{l}24 \text { months with } \\
\text { Echocardiography, } \\
\text { ECG, Holter } \\
\text { monitoring }\end{array}$ & $\begin{array}{l}\text { Increase in LVEF with LV } \\
\text { function improvements in } \\
\text { stem cell group }\end{array}$ \\
\hline $\begin{array}{l}\text { Srimahachota } \\
\text { et al. [30] }\end{array}$ & Thailand / 2011 & RCT & $\begin{array}{l}\mathrm{H} / \mathrm{o} \mathrm{STEMI} \text { with LVEF }<50 \% \\
\text { and PCI }\end{array}$ & 23 & Autologous $\mathrm{BMCs}=1$ & $\begin{array}{l}\text { Standard } \\
\text { therapy }=12\end{array}$ & $\begin{array}{l}\text { Six months with } \\
\text { cardiac MRI }\end{array}$ & $\begin{array}{l}\text { Symptoms improved than } \\
\text { baseline, but not many } \\
\text { significant changes were } \\
\text { noticed in the two groups }\end{array}$ \\
\hline
\end{tabular}




\section{Cureus}

\begin{tabular}{|c|c|c|c|c|c|c|c|c|}
\hline $\begin{array}{l}\text { Sürder et al. } \\
\text { [31] }\end{array}$ & $\begin{array}{l}\text { Switzerland / } \\
2013\end{array}$ & RCT & Acute MI & 200 & $\begin{array}{l}\text { BMMNC five-seven days } \\
\text { after STEMI = 66, BMMNC } \\
\text { three to four weeks after } \\
\text { STEMI }=67\end{array}$ & $\begin{array}{l}\text { Standard } \\
\text { therapy }=67\end{array}$ & $\begin{array}{l}\text { Four months with } \\
\text { cardiac MRI }\end{array}$ & No significant improvements \\
\hline $\begin{array}{l}\text { Traverse et } \\
\text { al. [32] }\end{array}$ & USA / 2012 & RCT & $\mathrm{MI}$ and $\mathrm{PCI}, \mathrm{LVEF}<45 \%$ & 120 & $\begin{array}{l}\text { BMMNC at day three or } \\
\text { day seven randomly = } 79\end{array}$ & Placebo $=41$ & $\begin{array}{l}\text { Six months with } \\
\text { cardiac MRI }\end{array}$ & No significant improvement \\
\hline $\begin{array}{l}\text { Traverse et } \\
\text { al. [33] }\end{array}$ & USA / 2011 & RCT & Acute $\mathrm{MI}$ and $\mathrm{PCl}$, LVEF $<45 \%$ & 87 & $\begin{array}{l}\text { BMMNC after two to three } \\
\text { weeks of } \mathrm{MI}=58\end{array}$ & Placebo $=29$ & $\begin{array}{l}\text { Six months with } \\
\text { cardiac MRI }\end{array}$ & No significant improvement \\
\hline $\begin{array}{l}\text { Traverse et } \\
\text { al. [34] }\end{array}$ & USA / 2018 & RCT & $\begin{array}{l}\text { Patients of TIME trial, acute MI } \\
\text { and } \mathrm{PCI}, \mathrm{LVEF}<45 \%\end{array}$ & 120 & $\begin{array}{l}\text { BMMNC at day three or } \\
\text { day seven randomly }=79 . \\
58 \text { patients were followed } \\
\text { up }\end{array}$ & $\begin{array}{l}\text { Placebo }=41 \text {, } \\
27 \text { patients } \\
\text { were followed } \\
\text { up }\end{array}$ & $\begin{array}{l}\text { Two years with } \\
\text { cardiac MRI }\end{array}$ & No significant improvement \\
\hline $\begin{array}{l}\text { Turan et al. } \\
\text { [35] }\end{array}$ & Germany / 2011 & RCT & $\begin{array}{l}\text { 18-80 years old with MI and LV } \\
\text { dysfunction }\end{array}$ & 56 & $\mathrm{BMMNC}=38$ & Placebo = 18 & $\begin{array}{l}\text { Three, six months, } \\
\text { and one year with } \\
\text { left } \\
\text { ventriculography }\end{array}$ & $\begin{array}{l}\text { Decrease in infarct size but } \\
\text { an increase of global EF and } \\
\text { infarct wall movement } \\
\text { velocity in stem cell group }\end{array}$ \\
\hline $\begin{array}{l}\text { Yerebakan et } \\
\text { al. [36] }\end{array}$ & Germany / 2011 & RCT & $\begin{array}{l}\text { MI at least } 14 \text { days before } \\
\text { admission and } \mathrm{LV} \text { akinesia with } \\
\text { an indication for CABG }\end{array}$ & 55 & $\begin{array}{l}\text { Intramyocardial CD133 (+) } \\
\text { BMCs }+ \text { CABG }=35\end{array}$ & $\begin{array}{l}\text { Only CABG = } \\
20\end{array}$ & $\begin{array}{l}18 \text { months with } \\
\text { 24-hour Holter } \\
\text { monitoring, } \\
\text { echocardiography, } \\
\text { MRI, and CT scan }\end{array}$ & $\begin{array}{l}\text { Intramyocardial stem cell } \\
\text { therapy was tolerable but did } \\
\text { not have significant } \\
\text { improvements }\end{array}$ \\
\hline
\end{tabular}

\section{TABLE 2: Characteristics and outcomes of the included studies}

STEMI = ST-Elevation Myocardial Infarction, $\mathrm{CAG}=$ Coronary Angiography, $\mathrm{PCI}=$ Percutaneous Coronary Intervention, $\mathrm{PPCl}=\mathrm{Primary}$ Percutaneous Coronary Intervention, LVEF = Left Ventricular Ejection Fraction, CABG = Coronary artery bypass grafting, CK = Creatine Kinase, H/o = History of, $\mathrm{MI}=$ Myocardial Infarction, CCT = Controlled Clinical Trial, CT = Clinical Trial, MNC = Mononuclear stem cells, CCTA = Coronary Computed Tomography Angiography, RCT = Randomized Controlled Trial, BMMNC = Bone Marrow Mononuclear Cell, BNP = B-type natriuretic peptide, WMSI = Wall Motion Score Index, N-BMC = Normoxia Bone Marrow Mononuclear Cells, HP-BMC = Hypoxia Preconditioned Bone Marrow Mononuclear Cells, ECG = Electrocardiogram, SPECT = Single-Photon Emission Computed Tomography, LV = Left Ventricle, MRI = Magnetic Resonance Imaging, CT scan = Computed Tomography scan, F-18-FDG-PET = F-18-Fluorodeoxyglucose Positron Emission Computed Tomography, 99mTc-SPECT = 99mTc-sestamibi Single-Photon Emission Computed Tomography, G-CSF = Granulocyte Colony-Stimulating Factor, MSCs = Mesenchymal Stem Cells, WJ-MSC = Wharton's jelly-derived Mesenchymal Stem Cells, CDC = Cardio sphere-derived autologous stem cell, LVEDD = End-Diastolic Dimension of the Left Ventricle, LVESV $=$ End-Systolic Volume of the Left Ventricle, LVEDV = End-Diastolic Volume of the Left Ventricle, SPCs = Stem/ Progenitor Cells, LIN- = Lineage-negative, CADUCEUS = CArdiosphere-Derived aUtologous stem CEIls to reverse ventricUlar dySfunction, TIME trial = Timing in Myocardial Infarction Evaluation trial

\section{Discussion}

After an acute myocardial infarction, patients usually suffer from left ventricular remodeling even after having successful revascularization [8]. Remodeling of the heart means changes in the size, shape, structure, and function of the cardiac muscles [37]. Our systematic review had 31 studies and 2171 patients. We observed the effectiveness of stem cells in an injured heart muscle after myocardial infarction.

\section{Effect of Stem Cell Therapy on Heart Function After Myocardial Infarction}

Benedek et al. conducted a controlled clinical trial (CCT). They included 18 patients in this trial, out of which nine patients received autologous bone marrow-derived mononuclear cells (BMMNC) [8]. On followup after four years, this study showed a slight improvement in ejection fraction (EF) in the stem cell group, the number of coronary plaques in segments infused with stem cell vs placebo group was ten vs twenty-one, calcium scoring in stem cell group vs placebo group was 295 vs 796, plaques creating $>50 \%$ stenosis in stem cell group vs placebo group were two vs eight and the plaque burden was much lower in coronary segments treated with stem cells [8].

Bozdag-Turan et al. conducted a prospective nonrandomized CCT in 24 patients in which he noticed a reduction in infarct size $(\mathrm{p}<0.001)$, an increase in global EF ( $\mathrm{p}=0.003)$, and an increase in infarct wall movement velocity. Additionally, B-type natriuretic peptide (BNP) level also decreased in the stem cell group $(\mathrm{p}<0.001)[10]$.

The clinical trial (CT) performed in 19 patients by Shah et al. demonstrated 12 patients who had received stem cell therapy, their echocardiography showed an increase in left ventricular ejection fraction (LVEF) from baseline at six months (3.8\%) which was sustained at two years (1.63\% increase), whereas in the control group LVEF was initially increased by $1.5 \%$ but at follow-up, in two years LVEF was decreased by 
Kim et al. concluded in their randomized clinical trial (RCT) of 26 patients that there was some improvement in LVEF [15]. Turan et al. in their RCT of 56 patients described there was a decrease in infarct size but an increase of global EF and infarct wall movement velocity in the stem cell group compared to the control group [35]. Similarly, Naseri et al. in their RCT of 77 patients noticed significant differences between the stem cell groups and placebo groups in LVEF and a decrease in the left ventricular (LV) thickening [23].

Gao et al. had 58 patients in their treatment group who had received $6 \times 10^{6}$ Wharton's Jelly-derived mesenchymal stem cells (WJ-MSC) dispersed in $10 \mathrm{~mL}$ heparinized saline and 58 patients on the control arm who received placebo [6]. 18 months later, follow-up revealed LVEF in the WJ-MSC group significantly increased in comparison to the placebo group. Also, left ventricular end-systolic and end-diastolic volumes were greatly decreased in the WJ-MSC group [6].

In the non-randomized CCT of Peregud-Pogorzelska et al. in 34 patients, they found that stem cell therapy is safe and $60 \%$ of patients from the bone marrow-derived lineage negative (LIN-) stem/progenitor cell group showed about > 10\% improvement in LVEF after a year with no signs of unfavorable remodeling of the left ventricle (LV) [25]. Similarly, one RCT conducted by Quyyumi et al. on 161 patients, among which 78 patients received an intracoronary infusion of autologous CD34 (+) cell (CLBS10) (cell therapy 10, Caladrius Biosciences Inc, Basking Ridge, NJ) revealed that stem cell therapy was safe and at one year, 3.6\% and $0 \%$ deaths were observed in the control and treatment group, respectively [26].

Additionally, the clinical trials conducted by Lee et al. and Micheu et al. found stem cell therapy is safe $[16,19]$.

Hu et al. in their RCT included 36 patients out of which 22 patients in the treatment arm either received normoxia-bone marrow cells (N-BMCs) or hypoxia-preconditioned bone marrow cells (HP-BMCs) and 14 patients received standard therapy [13]. There was an improvement in changes of left ventricular enddiastolic volume (LVEDV) and left ventricular end-systolic volume (LVESV) in HP-BMC group than N-BMC or control group ( $\mathrm{P}<0.05$ ), wall motion score index (WMSI) got better in HP-BMCs and N-BMC group $(\mathrm{P}<0.050)$, but not in the control group [13]. Additionally, the myocardial perfusion defect ratio was reduced in HP-BMCs and N-BMC groups at six months compared with baseline [13].

Makkar et al. conducted a CT in 25 patients, among which 17 patients in the control group were given cardio sphere-derived autologous stem cells; they showed at follow-up after six months [17] and one year [18] that the scar size was reduced, myocardial viability was increased along with the improved regional function of the damaged myocardium.

Roncalli et al. in their RCT included 52 patients receiving BMMNC and 49 patients receiving placebo [28]. Myocardial viability improved in 16/47 (34\%) patients in the treatment arm compared to $7 / 43$ (16\%) in the control group $(\mathrm{P}=0.06)$ and the number of non-viable segments becoming viable was $1.2 \pm 1.5$ in the BMMNC group and $0.8 \pm 1.1$ in the control group $(\mathrm{P}=0.13)$ [28]. At three months follow-up, the multivariate analysis showed improvement of myocardial viability than the univariate analysis $(P=0.03)[28]$. It also revealed that active smoking has a significant adverse effect $(\mathrm{P}=0.04)$, and a positive trend for microvascular obstruction $(\mathrm{P}=0.07)$ was observed as well [28].

Meanwhile, the double-blinded RCT of Alestalo et al. with 26 patients (14 receiving BMMNC and 12 receiving placebo) observed a harmonizing effect between the anti-inflammatory and pro-inflammatory cytokines in BMMNC treated ST-elevation myocardial infarction (STEMI) patients on day four [9]. The inflammation process of myocardial infarction (MI) was affected by this balancing effect, and it helped in remodeling and repair of the damaged heart muscles after an episode of acute MI [9].

In contrast, the double-blinded RCT of Choudry et al. in 100 patients showed although LVEF was increased compared with the baseline in both treatment and control groups, there was not much difference between the two groups $(2.2 \%$; $95 \%$ confidence interval, $\mathrm{CI}:-0.5$ to $5.0 ; \mathrm{P}=0.10)$ at one-year [11].

The RCT conducted by Nair et al. in 250 patients revealed, even though it is safe, stem cell therapy has no benefit in STEMI [22]. The number of patients in this study receiving the stem cell therapy deviated from 125 to 71 patients and the follow-up period was relatively short, which might have affected the outcome [22].

Similarly, Nicolau et al. in their RCT of 120 patients found intracoronary infusion of autologous bone marrow-derived mononuclear cells (BMMC) to STEMI patients did not improve LV function or decrease scar size [24]. This study did not have a core cell-processing laboratory, there was an unbalanced enrollment by the centers, and they used LVEF as the endpoint which may not be the most suitable endpoint to investigate the effect of cell infusion due to its constant changes in the acute phase [24]. These all factors had influenced the result. 
San Roman et al. in their RCT divided their study population into four groups which include one group of 30 patients receiving bone marrow mononuclear cells, 30 patients assigned to granulocyte colony-stimulating factor (G-CSF), 29 patients receiving G-CSF + cells, and a placebo group of 31 patients receiving standard therapy [29]. Patients treated with any of these stem cell approaches experienced similar changes in LVEF and LVESV when compared to the control group, with a small but significant reduction in infarct area $(\mathrm{p}=$ 0.038) [29]. One year later, cardiac magnetic resonance imaging (MRI) did not show much difference in these four groups [29]. But it was an open-labeled study and the study population was small which may have had an impact on the result of the study [29].

Srimahachota et al. concluded in their RCT in 23 patients that, stem cell therapy is safe but no improvement in LVEF can be described from the study [30]. The authors described a few reasons for not having a positive outcome such as the BMMNC cannot maintain at the infarcted area and a very few BMMNC remained at heart [30]. Also, cytokines may be needed to integrate the stem cells in the affected heart area to initiate the cells to trans-differentiate to cardiac myocyte, and the best cell type and timing for stem cell infusion is not yet known [30].

Similarly, Sürder et al. in their RCT of 200 patients [31] and Traverse et al. in their RCT of 120 patients explained that they did not notice any improvement in LV function in the stem cell group [32,34]. Traverse et al. demonstrated the use of cardiac MRI led to greater dropout of patients during the follow-up period because of device implantation in patients with more severe LV dysfunction which negatively impacted the outcome of their study [34].

Also, two CTs by Huang et al. and Rodrigo et al. showed there is no significant improvement in left ventricular function clinically [14,27]. The study of Rodrigo et al. was underpowered as they had a small number of patients and they used echocardiography rather than magnetic resonance imaging (MRI) to observe the effects on LV [27]. Even though single-photon emission computed tomography (SPECT) imaging showed an improvement in myocardial perfusion after three months of stem cell treatment, since SPECT imaging was not repeated in the control group, the effect of bone marrow-derived mesenchymal stem cells (MSC) therapy on myocardial perfusion can not be evaluated [27].

Two other RCTs focused on patients who received stem cells with coronary artery bypass grafting (CABG) $[12,36]$. Duan et al. had a treatment group of 24 patients having CABG + BMMNC and a control group of 18 patients who only underwent CABG [12]. One year post-surgery follow-up with echocardiography showed significant improvement in LV function including improvements in the end-diastolic dimension of the left ventricle (LVEDD), end-systolic dimension of the left ventricle (LVESD), LVEDV indexed to body surface area (LVEDVI), LVESV indexed to body surface area (LVESVI), the mass of left ventricle (LV-mass) and LV-mass indexed to body surface area (LV-mass I) compared to the data collected before the operation in CABG+BMMNC group [12].

Similarly, Yerebakan et al. had 35 patients who received intramyocardial CD133 (+) bone marrow stem cell transplant $+\mathrm{CABG}$, and 20 patients who only had CABG [36]. Follow-up after 18 months post-surgery showed intramyocardial stem cell therapy was well tolerated but did not have many significant improvements [36]. The authors mentioned that no follow-up angiography was performed, there was an unplanned withdrawal of patients which resulted in incomplete follow-up testing, and a limited number of patients were available for the final analysis [36]. In addition, MRI was not available in the preoperative assessment, so the results of this study should be accepted with caution [36].

Most of the mentioned studies agreed that there was a significant improvement in myocardial function, mainly the left ventricular end-diastolic volume and left ventricular end-systolic volume as well as ejection fraction after treatment with stem cell therapy. In addition, they showed no evidence of adverse effects in patients after receiving stem cell therapy.

However, some of the included studies reported there was no improvement or benefit from stem cell therapy in myocardial infarction treatment, even though stem cell therapy was safe and well-tolerable to those MI patients. The reason behind it is the studies were not done in a larger population and many patients were lost during follow-up. In addition to that, the optimum time for the administration of stem cells is not yet established.

So, from our systematic review, we can conclude that we need to perform more trials in a larger population and follow up with them closely to find out the effectiveness of stem cell therapy in patients with myocardial infarction.

Types of Stem Cells Used

One randomized clinical trial (RCT) performed by Gao et al. used Wharton's Jelly-derived mesenchymal stem cells (WJ-MSC) [6]. WJ-MSCs display more cardiovascular differentiation potential and as they are immune privileged, they can be transplanted into unrelated recipients [6]. 


\section{Cureus}

Another two studies used cardio sphere-derived autologous stem cells [17,18], whereas Naseri et al. in their RCT used CD133 (+) and mononuclear cells [23].

In the study of Rodrigo et al. bone marrow-derived mesenchymal stem cells (MSC), a subpopulation of bone marrow cells was used which can differentiate into several cell types including vascular cells, functional cardiomyocytes, etc [27]. In some preclinical models of acute myocardial infarction, it is seen that MSC transplantation promotes neovascularization and myogenesis, which in turn results in improved myocardial function [27].

San Roman et al. studied four groups which include one group of 30 patients receiving bone marrow mononuclear cells (BMMNC), 30 patients assigned to granulocyte colony-stimulating factor (G-CSF), 29 patients receiving G-CSF + BMMNCs, and a placebo group of 31 patients receiving standard therapy [29].

Quyyumi et al. in their RCT in 161 patients used autologous CD34 (+) cell (CLBS10) in 78 patients [26], whereas Peregud-Pogorzelska et al. delivered autologous bone marrow-derived lineage negative stem/progenitor cells in 15 patients in the treatment arm [25]. The researchers of the rest of our 24 included studies used autologous bone marrow mononuclear stem cells to treat the patients. We observed that the type of stem cell used did not have any influence on the outcome of this therapy.

Figure 3 given below illustrates the different types of stem cells used to regenerate the damaged heart muscle.
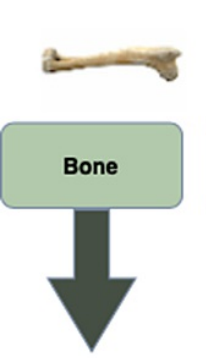
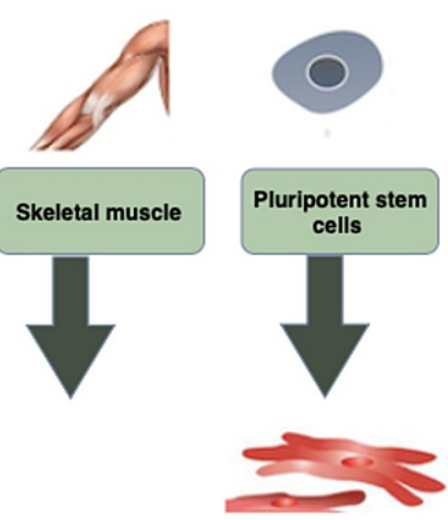

\section{Cardiomyocyte /} Progenitor cells
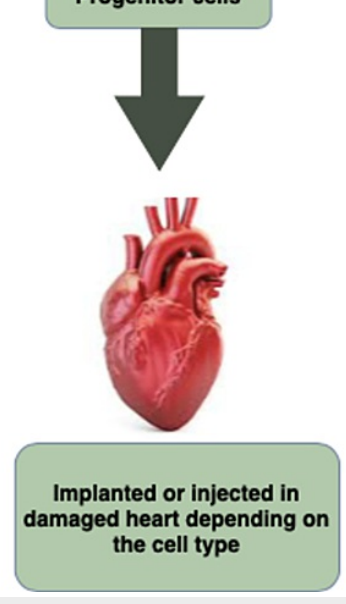

FIGURE 3: Different types of stem cells used to regenerate the damaged heart muscle

Route and Time of Administration of Stem Cell

Regarding the route of administration, intracoronary administration of the stem cell was performed in all our included studies. In addition to that, Moreira et al. in their randomized control trial (RCT) showed retrograde approach (intravenous coronary approach) to deliver stem cells was safe and cell retention by cardiac tissue is more in the anterograde (intra-arterial coronary) approach [21]. Timing of administration 
did not have much effect on the outcome. Huang et al. in their clinical trial (CT) divided the control group into three subgroups, and they got bone marrow mononuclear stem cells (BMMNC) infusion within two hours, three to seven days after percutaneous coronary intervention (PCI), and seven to thirty days after PCI respectively [14]. Effects of cell therapy given within 24 hours were noticed the same as given three to seven days after the primary PCI [14]. Similarly, Sürder et al. in their RCT demonstrated there is not much difference in the outcomes in groups where BMMNCs are administered five to seven days after ST-elevation myocardial infarction vs in groups where it is administered three to four weeks later [31].

Limitations

There are some limitations of our study, we had a small number of people used in these studies, and a few of them were lost during the follow-up. Additionally, the optimal time of stem cell delivery has not been determined. Moreover, we included only the studies conducted from 2011- 2021 to concentrate more on the updated information.

\section{Conclusions}

Our study focused on evaluating the safety and effectiveness of stem cell therapy in patients with acute myocardial infarction. We found that most of our included studies showed significant improvement in myocardial function after stem cell therapy, but some of the studies failed to show the same improvement. Also, we observed that the stem cell therapy was safe, well-tolerated and no major adverse effects were reported. Because the result was still inconsistent and contradictory, we need to perform high-quality, welldesigned clinical trials with a large sample size and more comparable results to assess and establish the efficacy of stem cell therapy in patients with acute myocardial infarction.

\section{Additional Information}

\section{Disclosures}

Conflicts of interest: In compliance with the ICMJE uniform disclosure form, all authors declare the following: Payment/services info: All authors have declared that no financial support was received from any organization for the submitted work. Financial relationships: All authors have declared that they have no financial relationships at present or within the previous three years with any organizations that might have an interest in the submitted work. Other relationships: All authors have declared that there are no other relationships or activities that could appear to have influenced the submitted work.

\section{References}

1. Kolandaiveloo L, Seghayat MS, Amini F: Efficacy and safety of autologous bone marrow cell therapy in treatment of acute myocardial infarction. Regen Res. 2016, 4:15-24.

2. Gerbin KA, Murry CE: The winding road to regenerating the human heart . Cardiovasc Pathol. 2015, 24:13340. 10.1016/j.carpath.2015.02.004

3. Shah VK, Shalia KK: Stem cell therapy for acute myocardial infarction-long term 24 months follow-up . J Clin Trials Cardiol. 2014, 1:1-5. 10.15226/2374-6882/1/2/00112

4. Carvalho E, Verma P, Hourigan K, Banerjee R: Myocardial infarction: stem cell transplantation for cardiac regeneration. Regen Med. 2015, 10:1025-43. 10.2217/rme.15.63

5. Ye J, Yeghiazarians Y: Cardiac stem cell therapy: review of the native cardiac progenitor cells and future direction. J Cardiovasc Pharmacol. 2014, 63:85-94. 10.1097/FJC.0b013e318299ebc0

6. Gao LR, Chen Y, Zhang NK, et al.: Intracoronary infusion of Wharton's jelly-derived mesenchymal stem cells in acute myocardial infarction: double-blind, randomized controlled trial. BMC Med. 2015, 13:162. 10.1186/s12916-015-0399-z

7. Strauer BE, Brehm M, Zeus T, et al.: Repair of infarcted myocardium by autologous intracoronary mononuclear bone marrow cell transplantation in humans. Circulation. 2002, 106:1913-18. 10.1161/01.cir.0000034046.87607.1c

8. Benedek I, Bucur O, Benedek T: Intracoronary infusion of mononuclear bone marrow-derived stem cells is associated with a lower plaque burden after four years. J Atheroscler Thromb. 2014, 21:217-29. 10.5551/jat.19745

9. Alestalo K, Miettinen JA, Vuolteenaho O, Huikuri H, Lehenkari P: Bone marrow mononuclear cell transplantation restores inflammatory balance of cytokines after ST segment elevation myocardial infarction. PLoS One. 2015, 10:e0145094. 10.1371/journal.pone.0145094

10. Bozdag-Turan I, Turan RG, Ludovicy S, et al.: Intra coronary freshly isolated bone marrow cells transplantation improve cardiac function in patients with ischemic heart disease. BMC Res Notes. 2012, 5:195. 10.1186/1756-0500-5-195

11. Choudry F, Hamshere S, Saunders N, et al.: A randomized double-blind control study of early intra-coronary autologous bone marrow cell infusion in acute myocardial infarction: the REGENERATE-AMI clinical trial $\dagger$. Eur Heart J. 2016, 37:256-63. 10.1093/eurheartj/ehv493

12. Duan F, Qi Z, Liu S, Lu X, Wang H, Gao Y, Wang J: Effectiveness of bone marrow mononuclear cells delivered through a graft vessel for patients with previous myocardial infarction and chronic heart failure: an echocardiographic study of left ventricular remodeling. Med Ultrason. 2015, 17:160-6. 10.11152/mu.2013.2066.172.effbm

13. Hu X, Huang X, Yang Q, et al.: Safety and efficacy of intracoronary hypoxia-preconditioned bone marrow mononuclear cell administration for acute myocardial infarction patients: The CHINA-AMI randomized 
controlled trial. Int J Cardiol. 2015, 184:446-51. 10.1016/j.ijcard.2015.02.084

14. Huang R, Yao K, Sun A, et al.: Timing for intracoronary administration of bone marrow mononuclear cells after acute ST-elevation myocardial infarction: a pilot study. Stem Cell Res Ther. 2015, 6:112. 10.1186/s13287-015-0102-5

15. Kim SH, Cho JH, Lee YH, et al.: Improvement in left ventricular function with intracoronary mesenchymal stem cell therapy in a patient with anterior wall ST-segment elevation myocardial infarction. Cardiovasc Drugs Ther. 2018, 32:329-38. 10.1007/s10557-018-6804-z

16. Lee JW, Lee SH, Youn YJ, et al.: A randomized, open-label, multicenter trial for the safety and efficacy of adult mesenchymal stem cells after acute myocardial infarction. J Korean Med Sci. 2014, 29:23-31. 10.3346/jkms.2014.29.1.23

17. Makkar RR, Smith RR, Cheng K, et al.: Intracoronary cardiosphere-derived cells for heart regeneration after myocardial infarction (CADUCEUS): a prospective, randomised phase 1 trial. Lancet. 2012, 379:895-904. 10.1016/S0140-6736(12)60195-0

18. Malliaras K, Makkar RR, Smith RR, et al.: Intracoronary cardiosphere-derived cells after myocardial infarction: evidence of therapeutic regeneration in the final 1-year results of the CADUCEUS trial (CArdiosphere-Derived aUtologous stem CElls to reverse ventricUlar dySfunction). J Am Coll Cardiol. 2014, 63:110-22. 10.1016/j.jacc.2013.08.724

19. Micheu MM, Oprescu N, Scarlatescu AI, Calmac L, Pitic D, Dorobanţu M: TRANSTEM pilot study-design, safety and results 6 months after autologous bone marrow stem cell transplant in patients with acute myocardial infarction and impaired left ventricular function. J Transl Med Res . 2015, 20:215-21.

20. Moccetti T, Sürder D, Klersy C, et al.: Sustained improvement in left ventricular function after bone marrow derived cell therapy in patients with acute ST elevation myocardial infarction. A 5-year follow-up from the stem cell transplantation in ischaemic myocardium study. Swiss Med Wkly. 2012, 142:w13632. 10.4414/smw.2012.13632

21. Moreira Rde C, Haddad AF, Silva SA, et al.: Intracoronary stem-cell injection after myocardial infarction: microcirculation sub-study. Arq Bras Cardiol. 2011, 97:420-6. 10.1590/s0066-782x2011005000095

22. Nair V, Madan H, Sofat S, et al.: Efficacy of stem cell in improvement of left ventricular function in acute myocardial infarction--MI3 Trial. Indian J Med Res. 2015, 142:165-74. 10.4103/0971-5916.164245

23. Naseri MH, Madani H, Ahmadi Tafti SH, et al.: COMPARE CPM-RMI trial: intramyocardial transplantation of autologous bone marrow-derived CD133+ cells and MNCs during CABG in patients with recent MI: a phase II/III, multicenter, placebo-controlled, randomized, double-blind clinical trial. Cell J. 2018, 20:267-77. 10.22074/cellj.2018.5197

24. Nicolau JC, Furtado RH, Silva SA, et al.: Stem-cell therapy in ST-segment elevation myocardial infarction with reduced ejection fraction: a multicenter, double-blind randomized trial. Clin Cardiol. 2018, 41:392-9. 10.1002/clc.22882

25. Peregud-Pogorzelska M, Przybycień K, Baumert B, et al.: The effect of intracoronary infusion of autologous bone marrow-derived lineage-negative stem/progenitor cells on remodeling of post-infarcted heart in patient with acute myocardial infarction. Int J Med Sci. 2020, 17:985-94. 10.7150/ijms.42561

26. Quyyumi AA, Vasquez A, Kereiakes DJ, et al.: PreSERVE-AMI: a randomized, double-blind, placebocontrolled clinical trial of intracoronary administration of autologous CD34+ cells in patients with left ventricular dysfunction post STEMI. Circ Res. 2017, 120:324-31. 10.1161/CIRCRESAHA.115.308165

27. Rodrigo SF, van Ramshorst J, Hoogslag GE, et al.: Intramyocardial injection of autologous bone marrowderived ex vivo expanded mesenchymal stem cells in acute myocardial infarction patients is feasible and safe up to 5 years of follow-up. J Cardiovasc Transl Res. 2013, 6:816-25. 10.1007/s12265-013-9507-7

28. Roncalli J, Mouquet F, Piot C, et al.: Intracoronary autologous mononucleated bone marrow cell infusion for acute myocardial infarction: results of the randomized multicenter BONAMI trial. Eur Heart J. 2011, 32:1748-57. 10.1093/eurheartj/ehq455

29. San Roman JA, Sánchez PL, Villa A, et al.: Comparison of different bone marrow-derived stem cell approaches in reperfused STEMI. A multicenter, prospective, randomized, open-labeled TECAM trial. J Am Coll Cardiol. 2015, 65:2372-82. 10.1016/j.jacc.2015.03.563

30. Srimahachota S, Boonyaratavej S, Rerkpattanapipat $\mathrm{P}$, et al.: Intra-coronary bone marrow mononuclear cell transplantation in patients with ST-elevation myocardial infarction: a randomized controlled study. J Med Assoc Thai. 2011, 94:657-63.

31. Sürder D, Manka R, Lo Cicero V, et al.: Intracoronary injection of bone marrow-derived mononuclear cells early or late after acute myocardial infarction: effects on global left ventricular function. Circulation. 2013, 127:1968-79. 10.1161/CIRCULATIONAHA.112.001035

32. Traverse JH, Henry TD, Pepine CJ, et al.: Effect of the use and timing of bone marrow mononuclear cell delivery on left ventricular function after acute myocardial infarction: the TIME randomized trial. JAMA. 2012, 308:2380-9. 10.1001/jama.2012.28726

33. Traverse JH, Henry TD, Ellis SG, et al.: Effect of intracoronary delivery of autologous bone marrow mononuclear cells 2 to 3 weeks following acute myocardial infarction on left ventricular function: the LateTIME randomized trial. JAMA. 2011, 306:2110-9. 10.1001/jama.2011.1670

34. Traverse JH, Henry TD, Pepine CJ, et al.: TIME Trial: effect of timing of stem cell delivery following STelevation myocardial infarction on the recovery of global and regional left ventricular function: final 2-year analysis. Circ Res. 2018, 122:479-88. 10.1161/CIRCRESAHA.117.311466

35. Turan RG, Bozdag-T I, Ortak J, et al.: Improved functional activity of bone marrow derived circulating progenitor cells after intra coronary freshly isolated bone marrow cells transplantation in patients with ischemic heart disease. Stem Cell Rev Rep. 2011, 7:646-56. 10.1007/s12015-010-9220-8

36. Yerebakan C, Kaminski A, Westphal B, et al.: Impact of preoperative left ventricular function and time from infarction on the long-term benefits after intramyocardial CD133(+) bone marrow stem cell transplant. J Thorac Cardiovasc Surg. 2011, 142:1530-9.e3. 10.1016/j.jtcvs.2011.05.002

37. Azevedo PS, Polegato BF, Minicucci MF, Paiva SA, Zornoff LA: Cardiac remodeling: concepts, clinical impact, pathophysiological mechanisms and pharmacologic treatment. Arq Bras Cardiol. 2016, 106:62-9. 10.5935/abc. 20160005 